\title{
Dietary reference intakes: aplicabilidade das tabelas em estudos nutricionais
}

\author{
Dietary reference intakes: application of \\ tables in nutritional studies
}

\author{
Renata Maria PADOVANI' \\ Jaime AMAYA-FARFÁN' \\ Fernando Antonio Basile COLUGNATI2 \\ Semíramis Martins Álvares DOMENE ${ }^{3}$
}

\section{RE S U M O}

As avaliações de dietas e o planejamento de consumo são atividades tradicionalmente realizadas por meio da comparação de médias de ingestão contra valores de referência de energia e nutrientes, seja para indivíduos ou grupos. Limitações de ordem técnica devem ser levadas em conta, sem as quais se pode chegar a conclusões equivocadas quanto ao atendimento das necessidades nutricionais. As Recomendações Nutricionais propostas pelo Institute of Medicine dos Estados Unidos, em conjunto com a agência Health Canada, a partir de 1997, conhecidas como Dietary Reference Intakes, representam um novo paradigma para o estabelecimento de indicadores nutricionais de consumo, ao aperfeiçoarem o uso do conceito de risco na avaliação de dietas. Fontes de erro intra ou interindividuais, devidas à variabilidade de padrão de consumo e decorrentes da distribuição das necessidades na população, aliadas a um pequeno número de dias de observação, têm grande impacto sobre a confiabilidade da análise. Por esta razão devem orientar a utilização dos valores, que foram organizados em tabelas com as quatro categorias de nutrientes, publicadas entre 1997 e 2005 . O presente trabalho teve por objetivo destacar algumas características de aplicação e consolidar os valores diários de Tolerable Upper Intake Level, Adequate Intake e Recommended Dietary Allowance, facilitando a consulta por parte de profissionais e estudantes da área de nutrição.

Termos de indexação: avaliação nutricional; nutrientes; planejamento alimentar; recomendações nutricionais.

\section{A B S T R A C T}

Diet planning and food intake evaluation are professional activities classically performed by comparing mean values of ingestion against reference values of nutrient and energy intakes, for either groups or individuals.

\footnotetext{
1 Departamento de Alimentos e Nutrição, Faculdade de Engenharia de Alimentos, Universidade Estadual de Campinas. Av. Albert Einstein, 291, Cidade Universitária Zeferino Vaz, 13083-852, Campinas, SP, Brasil. Correspondência para/Correspondence to: R.M. PADOVANI. E-mail: <renatapadovani@uol.com.br>.

2 Núcleo de Estudos e Pesquisas em Alimentação, Universidade Estadual de Campinas. Campinas, SP, Brasil.

${ }^{3}$ Faculdade de Nutrição, Centro de Ciências da Vida, Pontifícia Universidade Católica de Campinas. Campinas, SP, Brasil.
} 
Without considering certain intrinsic limitations of the reference values, such task may render equivocal conclusions with regard to nutritional adequacy. The nutritional recommendations, prepared as a joint effort between the US Institute of Medicine and Health Canada published since 1997 and known as Dietary Reference Intakes, offer a new model by establishing indices of nutrient intake and refining the concept of risk associated with diet evaluation. Errors of both intra and interindividual nature, due to the variability of an individual's feeding pattern, the uneven distribution of requirements within a population, respectively, and the small number of days of observation, are factors that together can have a large impact on the confidence of the analysis. For these reasons, only the judicious selection of the reference values, published in the form of tables from 1997 through 2005, should enable the user to reach the proper assessment. This paper had the objective of highlighting various critical characteristics of application and compiling the values in order to facilitate their use by both professionals and students of the area.

Indexing terms: nutrition assessment; nutrients; food planning; nutritional policy.

\section{N T R O D U Ç Ã O}

Na análise da qualidade nutricional da alimentação e da programação de dietas considera-se o atendimento às necessidades de nutrientes e energia, determinadas de acordo com as características de sexo, estágio de vida, atividade física e medidas corporais de indivíduos saudáveis ${ }^{1-3}$.

Tanto para a avaliação da dieta como para sua prescrição, são estabelecidos valores de referência para ingestão de nutrientes, os quais são periodicamente revisados à luz de novos achados. Assim, são incorporados novos conhecimentos sobre eventuais manifestações aos extremos de exposição, ou seja, sinais carenciais decorrentes de ingestão insuficiente ${ }^{4}$, ou de toxicidade, que indicam efeitos adversos decorrentes do excesso de consumo ${ }^{5,6}$.

As Dietary Reference Intakes (DRI) constituem-se na mais recente revisão dos valores de recomendação de nutrientes e energia adotados pelos Estados Unidos e Canadá, e vêm sendo publicadas desde 1997, na forma de relatórios parciais elaborados por comitês de especialistas organizados por uma parceria entre o Institute of Medicine norte-americano e a agência Health Canada. Essas publicações substituem as sucessivas versões das Recommended Dietary Allowances (RDA), cuja décima revisão foi editada em 1989.

Além da atualização de cotas dietéticas recomendadas, esse conjunto de publicações apresenta um novo sistema de aplicação das quatro categorias de valores de referência para avaliação e planejamento de consumo, rotulagem e fortificação de alimentos. Os novos conceitos foram elaborados a partir da incorporação dos achados sobre o aumento dos riscos de desenvolvimento de doenças crônicas não transmissíveis, provocado pela alimentação, além da abordagem clássica sobre os efeitos de carência ${ }^{7,8}$. Estas categorias são:

- Estimated Average Requirement (EAR), este valor de referência corresponde à mediana da distribuição das necessidades de um nutriente em um grupo de indivíduos saudáveis do mesmo sexo e estágio de vida; por essa razão, atende às necessidades de $50 \%$ da população.

- Recommended Dietary Allowances (RDA), esta categoria de valores, já empregada nas versões anteriores, deriva do EAR e deve atender às necessidades de um nutriente para $97 \%$ a $98 \%$ dos indivíduos saudáveis do mesmo sexo e estágio de vida.

- Adequate Intake (Al), valor de consumo recomendável, baseado em levantamentos, determinações ou aproximações de dados experimentais, ou ainda de estimativas de ingestão de nutrientes para grupo(s) de pessoas sadias e que, a priori, se consideraria adequado. Nem sempre o conjunto de informações sobre o nutriente é suficientemente consistente para o estabelecimento de EAR. Nesses casos, deve-se empregar o valor de Al, projetado como possivelmente superior ao valor de RDA, mas sobre o qual ainda há considerável incerteza. Assim, o valor de Al é usado quando os valores de EAR ou de RDA não podem ser determinados. 
- Tolerable Upper Intake Level (UL), há, em alguns casos, a compreensão equivocada de que se um nutriente faz bem em pequena quantidade, uma grande quantidade traria proporcionalmente mais benefícios. De fato, nutrientes podem ser nocivos em doses que às vezes são apenas pouco superiores aos valores de recomendação. O UL é definido como o mais alto valor de ingestão diária prolongada de um nutriente que, aparentemente, não oferece risco de efeito adverso à saúde em quase todos os indivíduos de um estágio de vida ou sexo.

Entre as novidades desse sistema não estão apenas as quatro categorias de referência já mencionadas. Com as DRI pode-se aprimorar a avaliação e o planejamento da alimentação, ao considerar que, para cada uma dessas etapas da atenção dietética, deve-se aplicar uma ou mais das categorias citadas, de maneiras distintas.

Assim, para indivíduos, EAR e UL são as categorias de referência mais adequadas para a avaliação de dietas, enquanto RDA ou Al devem ser utilizadas como metas de ingestão. Valores habituais de consumo abaixo do EAR denotam grande probabilidade de inadequação, e acima do UL, risco de desenvolvimento de efeitos adversos. Contudo, se o consumo habitual estiver acima dos valores da RDA há maior chance de que as necessidades nutricionais, tanto de indivíduos quanto de populações, estejam atendidas.

Quando não há valor de EAR e apenas o valor de Al se encontra disponível, há maior incerteza para avaliar se um determinado nutriente é fornecido pela dieta em quantidade adequada. Portanto, pela simples aplicação das tabelas não é possível chegar a uma conclusão sobre inadequação, quando os valores de consumo habitual forem menores do que este valor de referência ${ }^{7-9}$.

Fontes de erro intraindividuais, devidas à variabilidade do padrão de consumo, ou interindividuais, decorrentes da distribuição das necessidades na população, aliadas a um pequeno número de dias de observação, têm grande impacto sobre a confiabilidade da análise. A adoção de técnicas adequadas de inquérito dietético, aplicadas duas ou mais vezes, preferencialmente em dias não consecutivos, contribui para melhorar a acurácia de métodos quantitativos de consumo ${ }^{10}$.

Outros avanços do sistema incluem a proposição do conceito de 'estágios da vida', e não mais 'faixas etárias'; a definição de antioxidante alimentar; o emprego do conceito de unidades equivalentes para vitamina $\mathrm{E}^{9}$ e folato ${ }^{11}$, além da vitamina $A^{12}$.

Adicionalmente, houve a inclusão de um maior número de elementos, como arsênico, boro, níquel e vanádio. Para estes, entretanto, a insuficiência das evidências para firmar suas funções sobre a saúde impede, até a presente data, o estabelecimento de níveis de recomendação ${ }^{12,13}$.

\section{Aplicação: desenvolvendo o conceito de risco}

As Dietary Reference Intakes incorporam, nesta edição, o conceito de risco, visto como medida de incerteza originada pelas fontes de variabilidade decorrentes de qualquer levantamento dietético. Tanto na abordagem individual, quanto na estimativa de prevalências de carência no âmbito populacional, a resposta se dá por meio de riscos, ou probabilidades, de adequação ou inadequação.

Para estudos de consumo por grupos populacionais, a proposta parte do conceito da 'necessidade' e da 'ingestão' do nutriente. Teoricamente, as distribuições de probabilidade para esses dois parâmetros deveriam ser conhecidas. Logo, o 'risco' é entendido como a chance de a população não ter suas necessidades nutricionais atendidas.

Conhecer a ingestão de nutrientes e energia por um grupo já implica em trabalho árduo 
em que se utilizam métodos com diversas fontes e graus de variabilidade ${ }^{14}$. Portanto, estimar a necessidade torna-se quase inviável na prática, sendo que a abordagem simplificada, baseada nos valores de EAR como pontos de corte, fornece estimativas mais seguras para o gerenciamento na esfera da saúde pública.

As premissas para a aplicação desse método nem sempre são de fácil verificação ${ }^{14}$. São elas: a) a 'necessidade' e a 'ingestão' são variáveis independentes; b) a distribuição das necessidades é 'simétrica' em torno da EAR, o que é distinto de distribuição 'normal', e c) a variabilidade da 'ingestão' é maior que a da 'necessidade'.

Recomenda-se também o uso desse método simplificado quando for possível assumir que as prevalências de inadequação em estudo sejam maiores do que $10 \%$ e menores do que $90 \%$. Com base nessas suposições, a prevalência estimada é dada pela proporção de indivíduos com ingestão abaixo da EAR. Caso as premissas não sejam satisfeitas pode haver super ou subestimativa dessa prevalência ${ }^{15}$. Vale ressaltar que a distribuição da EAR a ser utilizada, ou seja, as estimativas de ingestão da população estudada devem refletir apenas a variabilidade intra-individual, o que pode ser determinado a partir de métodos de ajustes ${ }^{10,16}$ sobre dados provenientes de inquéritos dietéticos de, pelo menos, dois ou três dias.

A aplicação das Dietary Reference Intakes para a avaliação e planejamento de dietas tem sido discutida na literatura nacional e não será detalhada aqui14,17,18. A presente compilação dos valores diários de UL, Al e RDA teve por objetivo facilitar a consulta por parte dos profissionais e estudantes da área de nutrição. Uma compilação anterior se referia aos nutrientes com características antioxidantes ${ }^{17}$.

As Tabelas 1 a 7 contêm os valores de referência das quatro categorias publicadas entre 1997 e 2005. Foram reunidos os valores de EAR e RDA (ou Al* , em negrito e com asterisco - notação dos autores para evitar confusão entre as diferentes fontes), além dos valores de UL. Observar que $1 \mu \mathrm{g}$ (micrograma) é a milésima parte de $1 \mathrm{mg}$ (miligrama) e 1 ng (nanograma) é a milésima parte de $1 \mu \mathrm{g}$. Dietary Reference Intakes e Adequate Intake podem ser usadas indistintamente como metas de ingestão para indivíduos. Recommended Dietary Allowances foram estabelecidas para atender à necessidade de praticamente todos (97\% a 98\%) os indivíduos do mesmo sexo e estágio de vida. Para bebês saudáveis em aleitamento exclusivo, $\mathrm{Al}$ é a ingestão média. Os valores de Al para os demais estágios de vida e ambos os gêneros devem atender às necessidades de todos os indivíduos no grupo, mas a incerteza sobre os dados disponíveis não permite estabelecer com confiança o percentual de indivíduos atendidos por esses valores. O emprego desses dados deve obedecer à metodologia proposta pelo Institute of Medicine, especialmente para ações de avaliação e planejamento de consumo por grupos. 


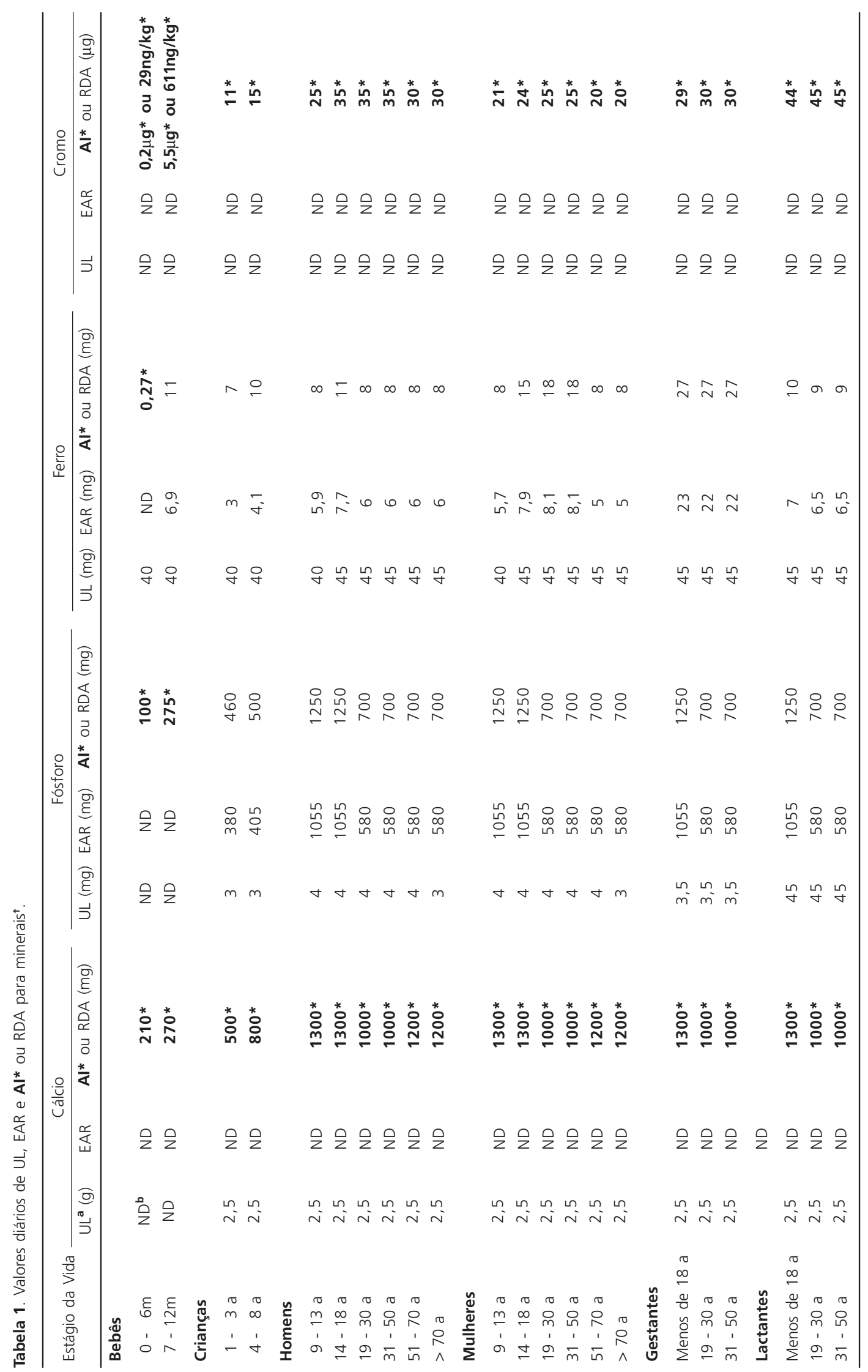


746 | R.M. PADOVANI et al.

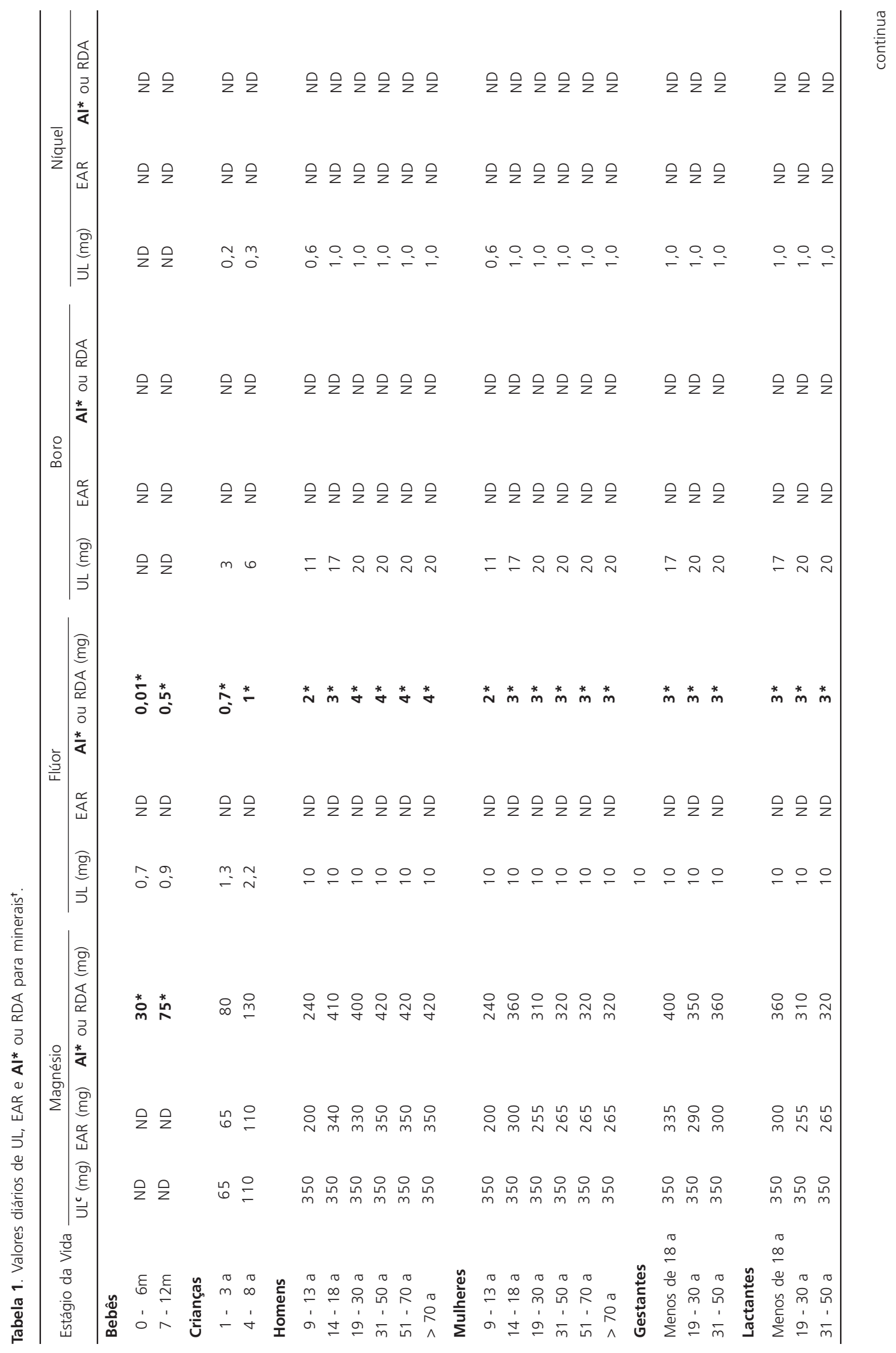


TABELAS E APLICABILIDADE DAS DRI | 747

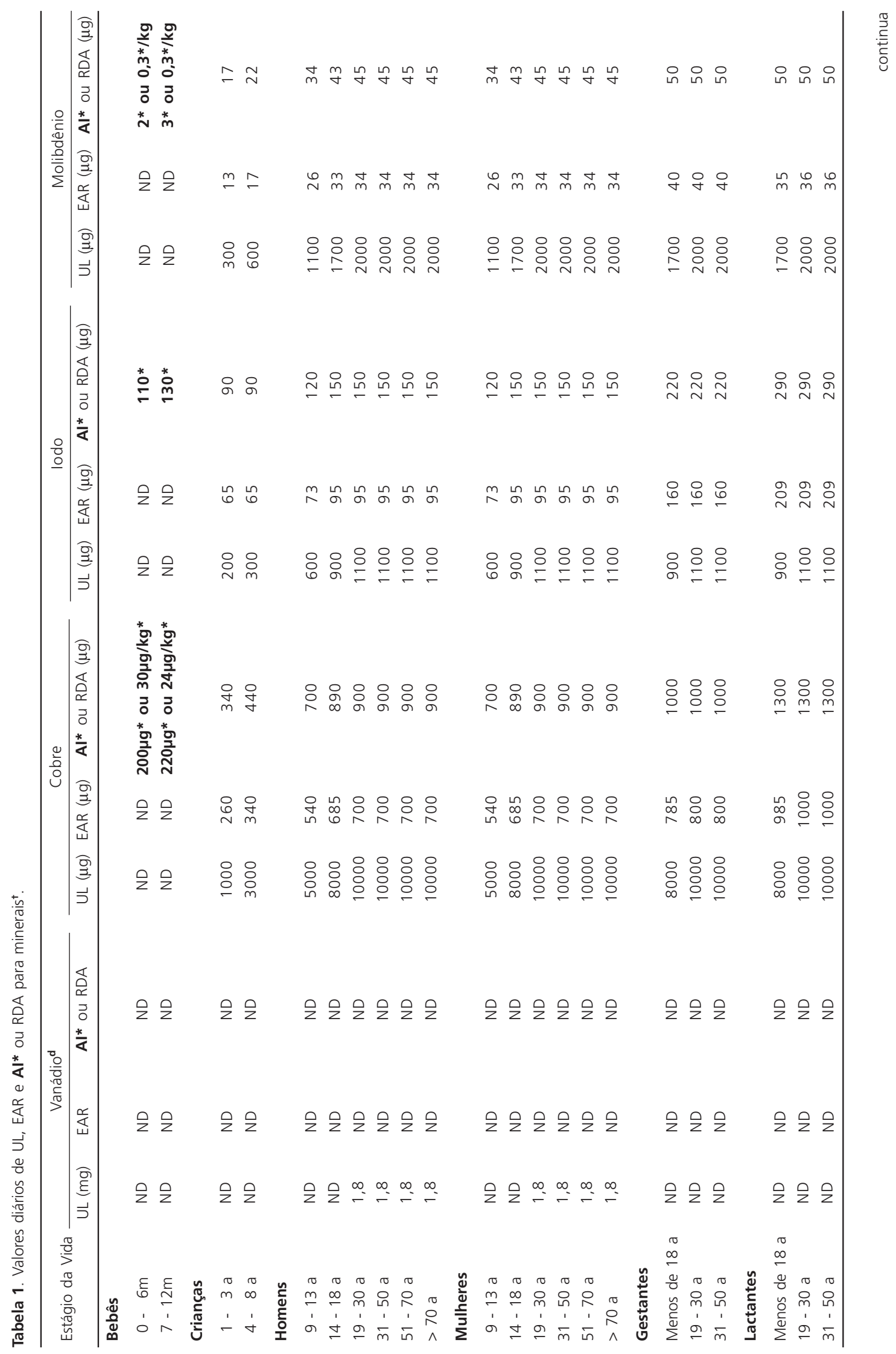


748 | R.M. PADOVANI et al.

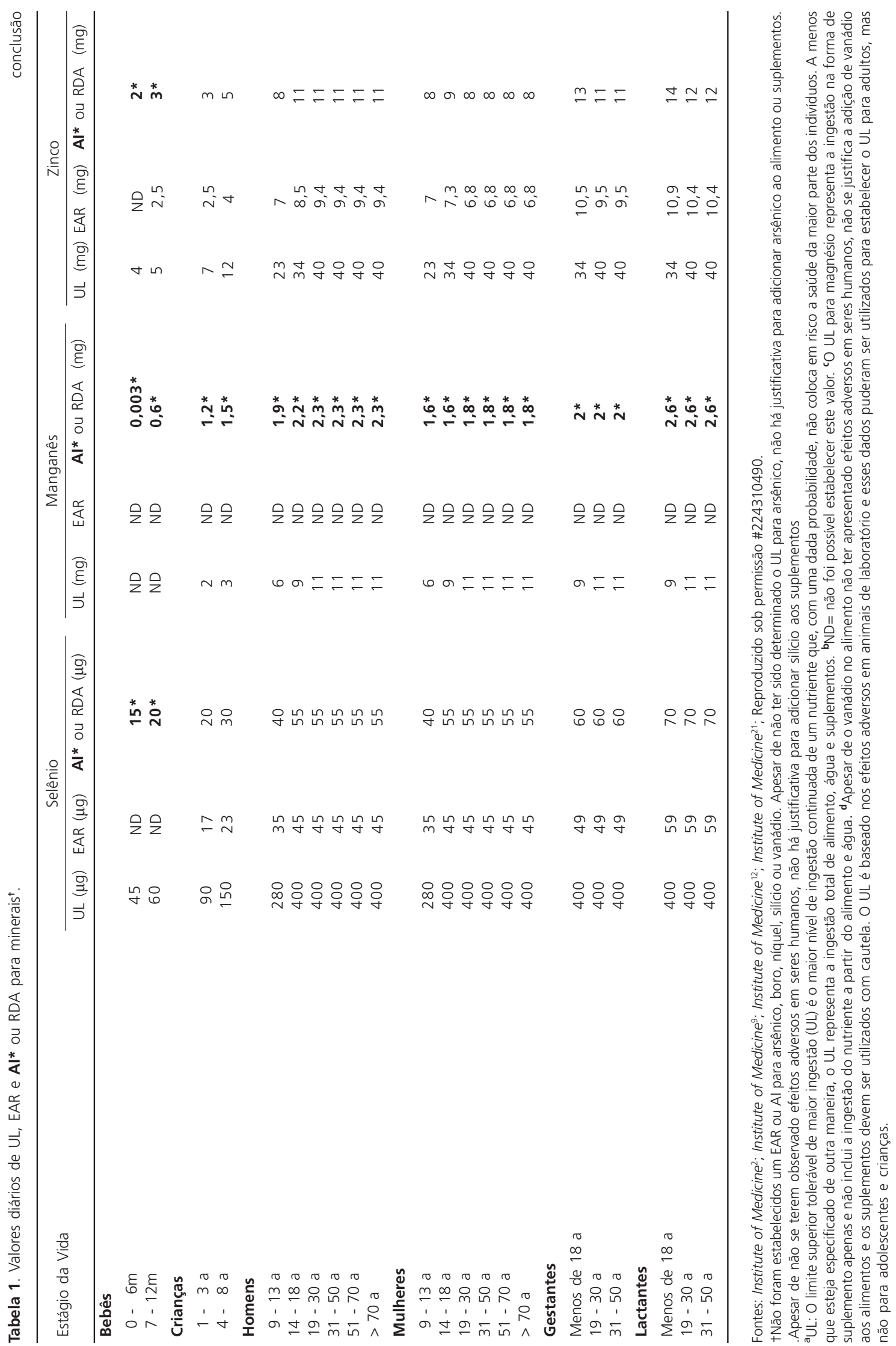




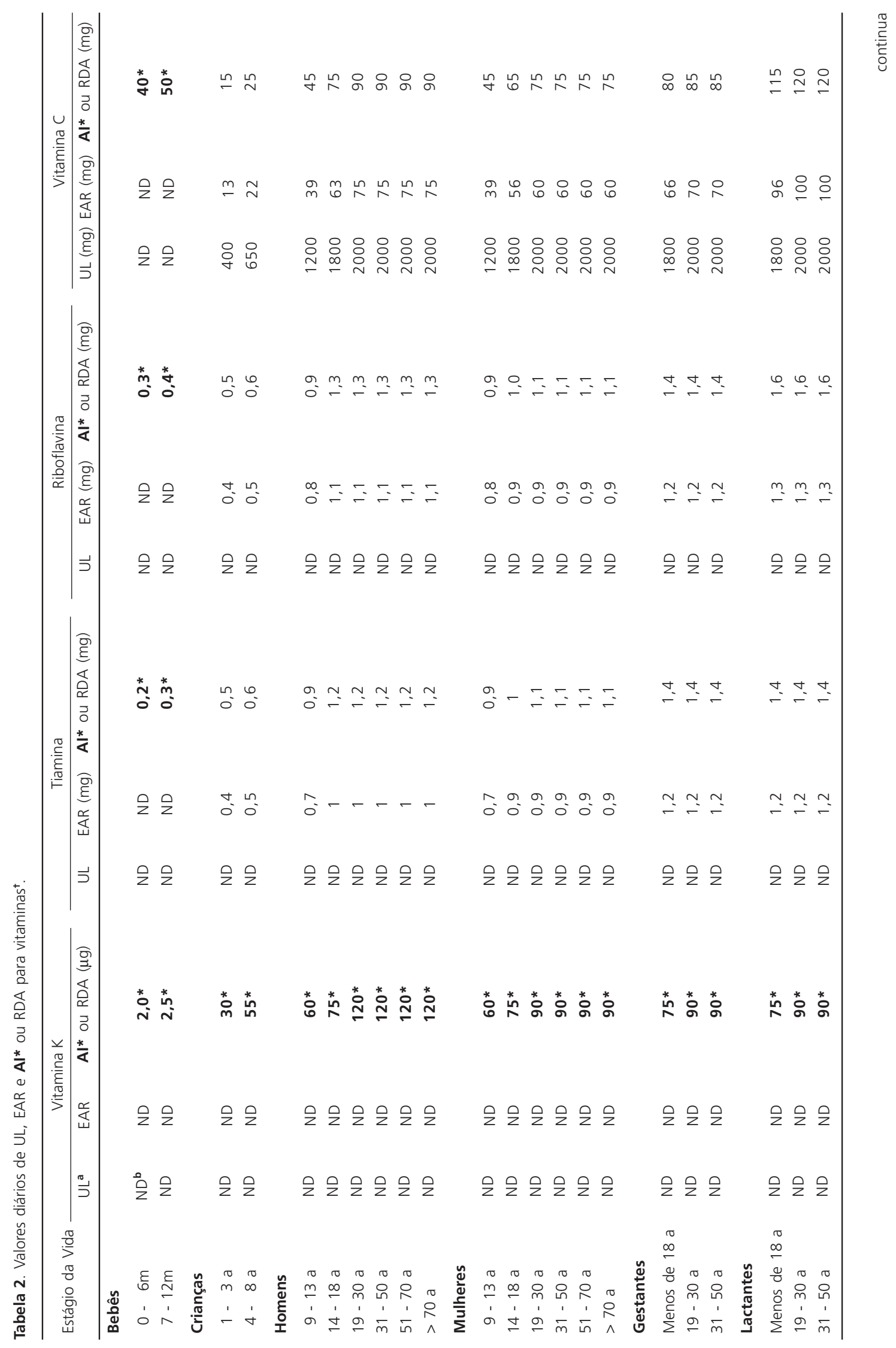


$750 \mid$ R.M. PADOVAN let al.

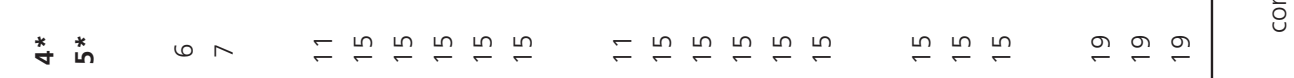

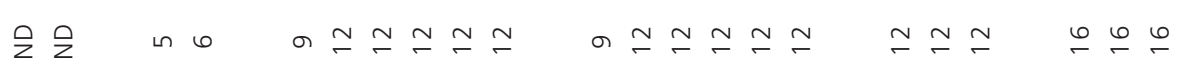

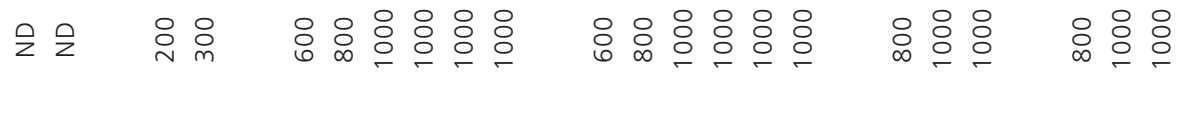

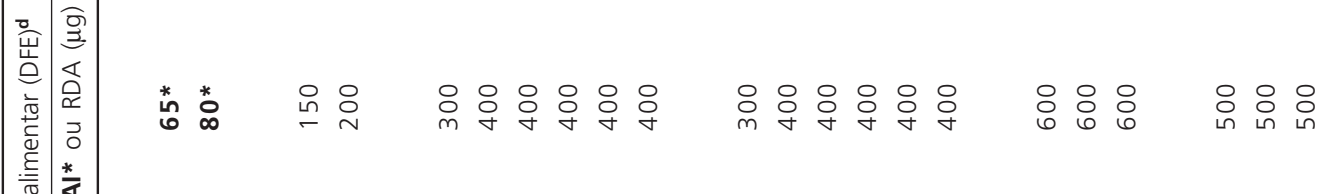

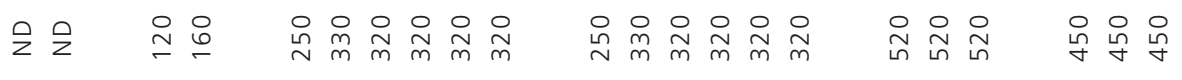

₹

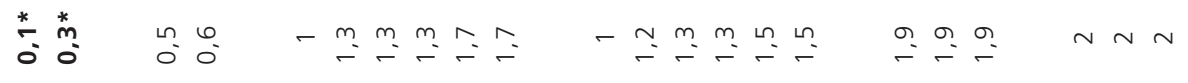

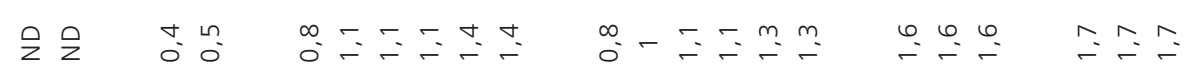

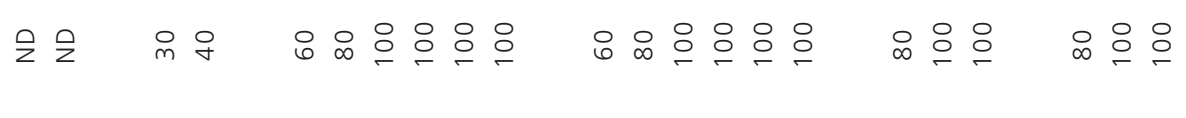

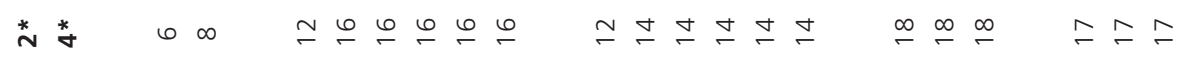

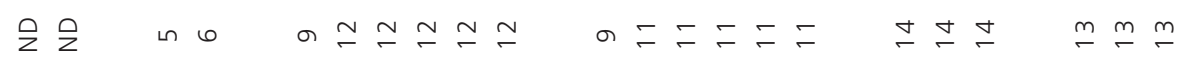

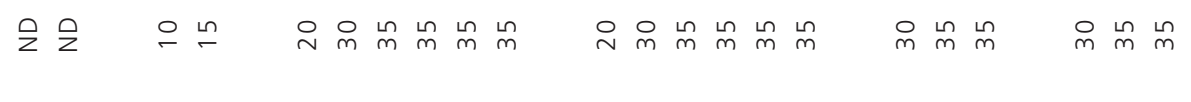

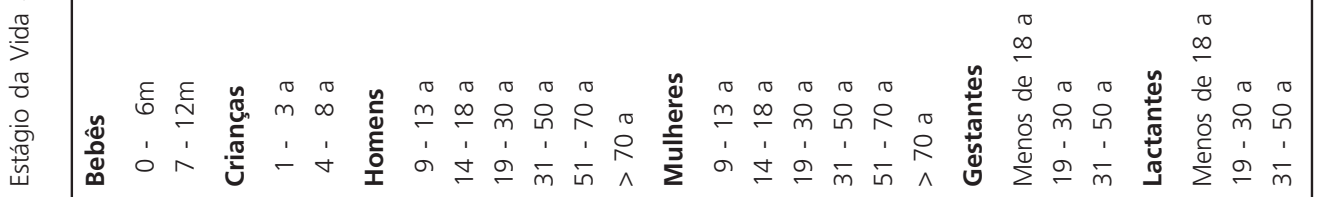




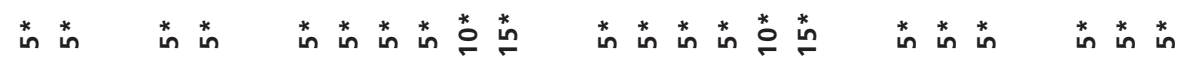

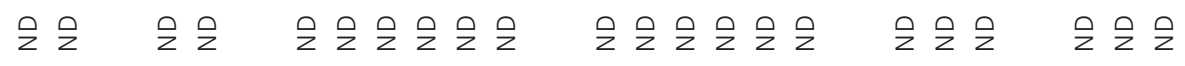

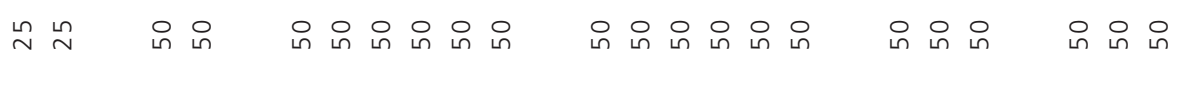

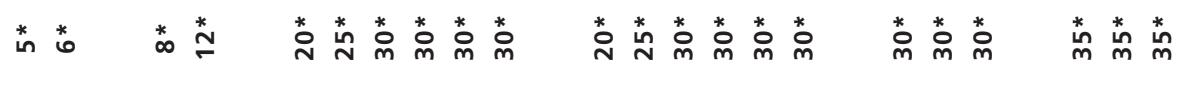

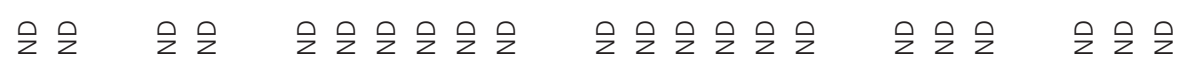

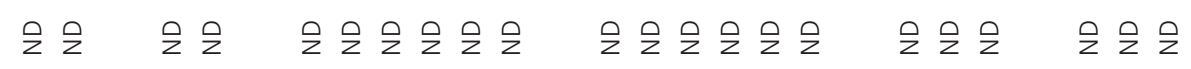
$\begin{array}{llllll}2 z z z z z z z z & z z z\end{array}$

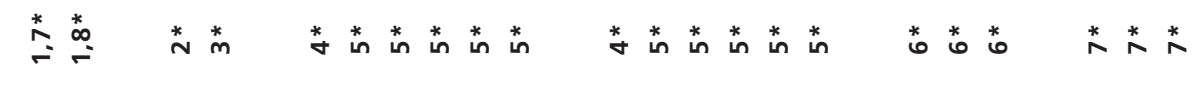

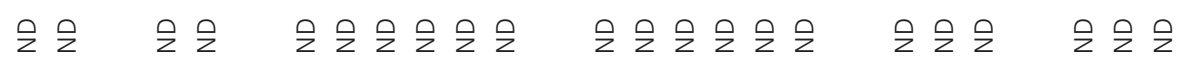

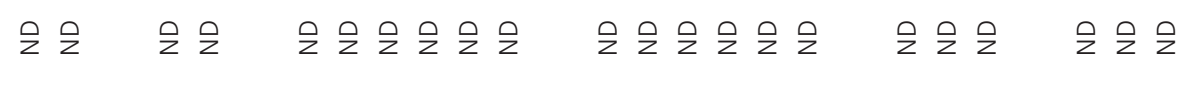

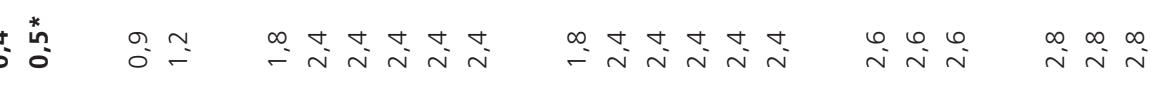

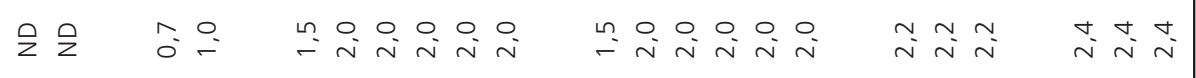

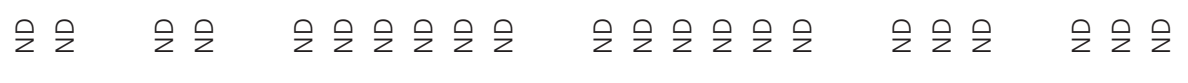


752 R.M. PADOVANI et al.

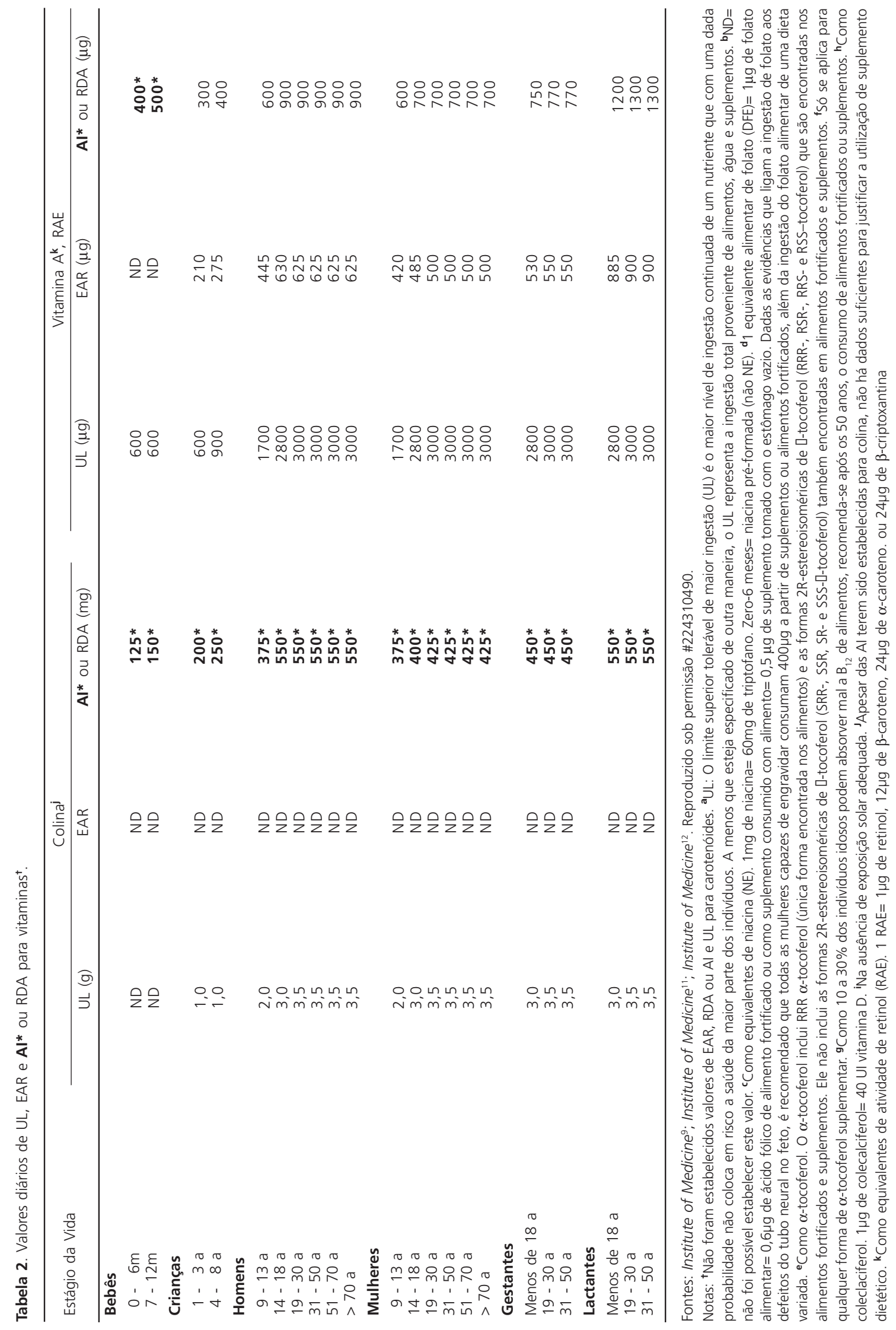


TABELAS E APLICABILIDADE DAS DRI | 753

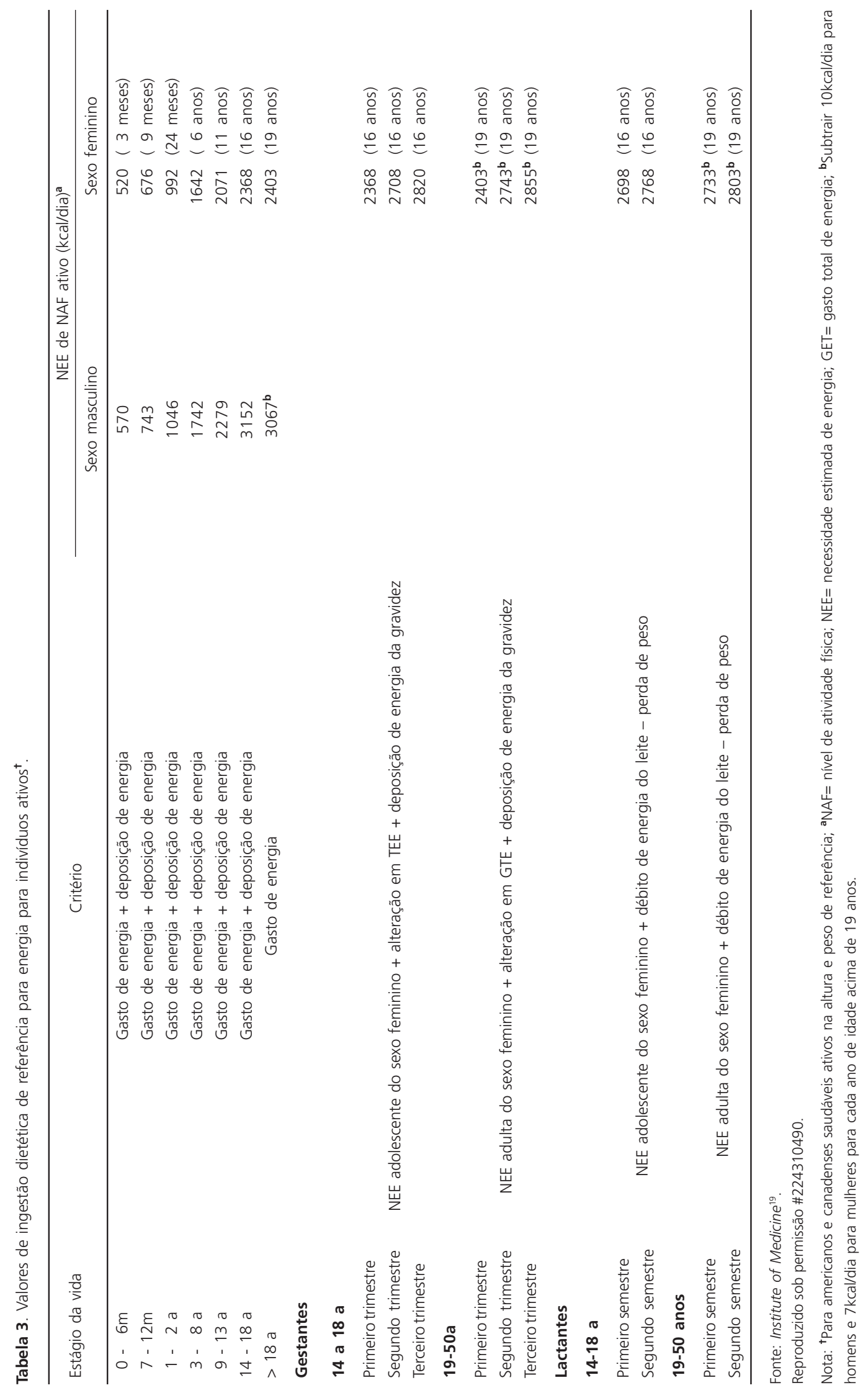




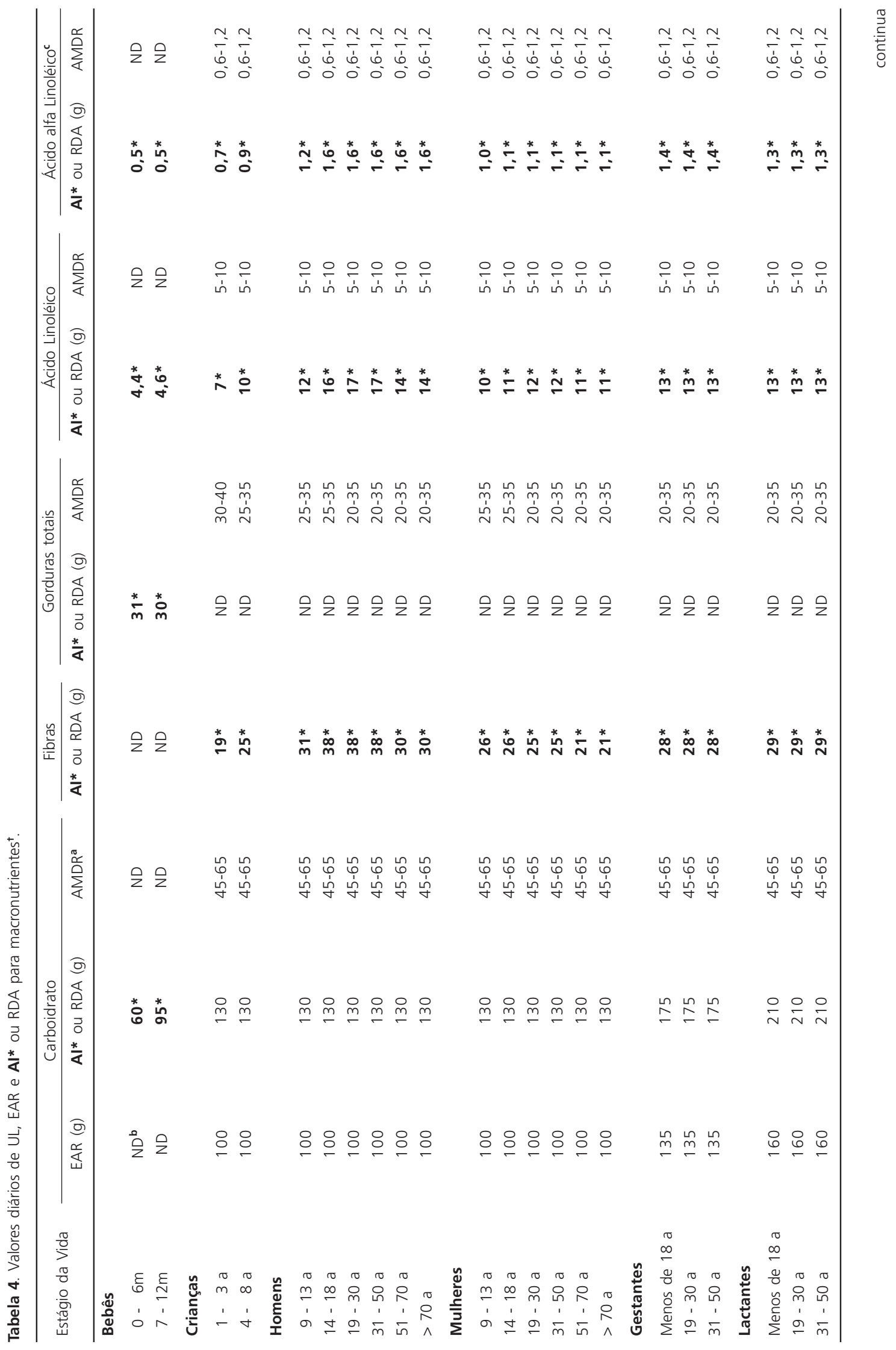




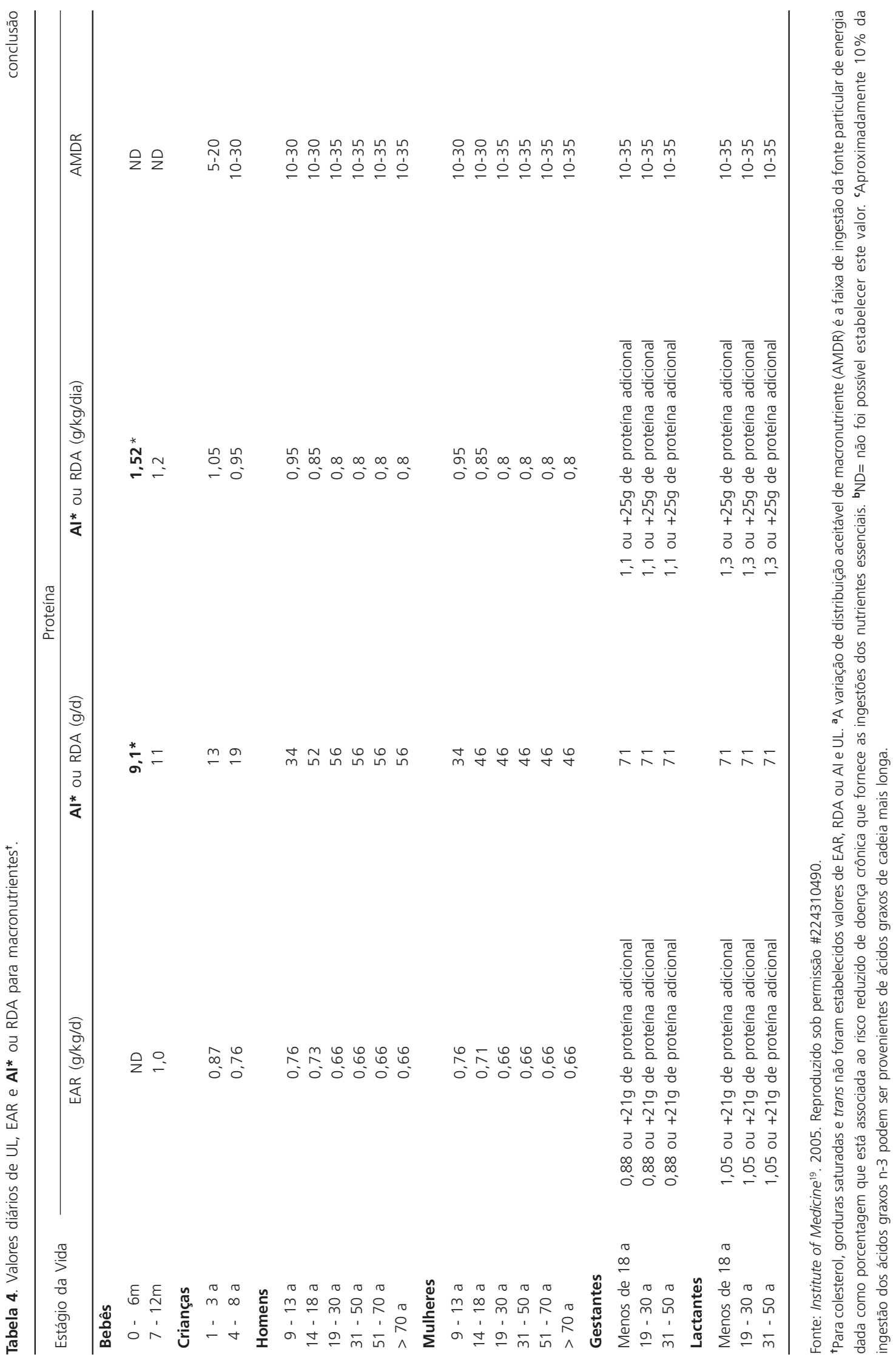


756 | R.M. PADOVAN et al.

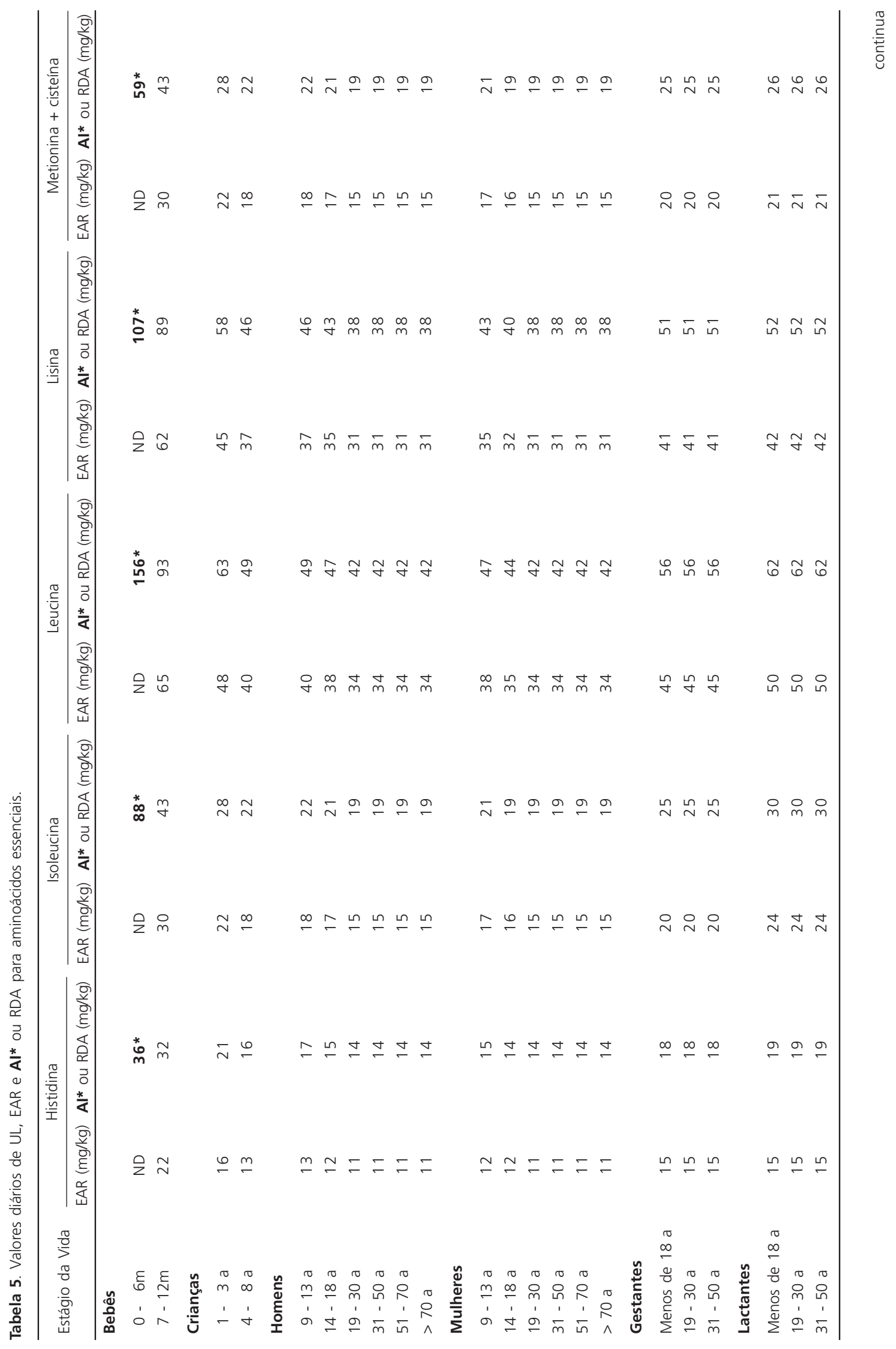


TABELAS E APLICABILIDADE DAS DRI | 757

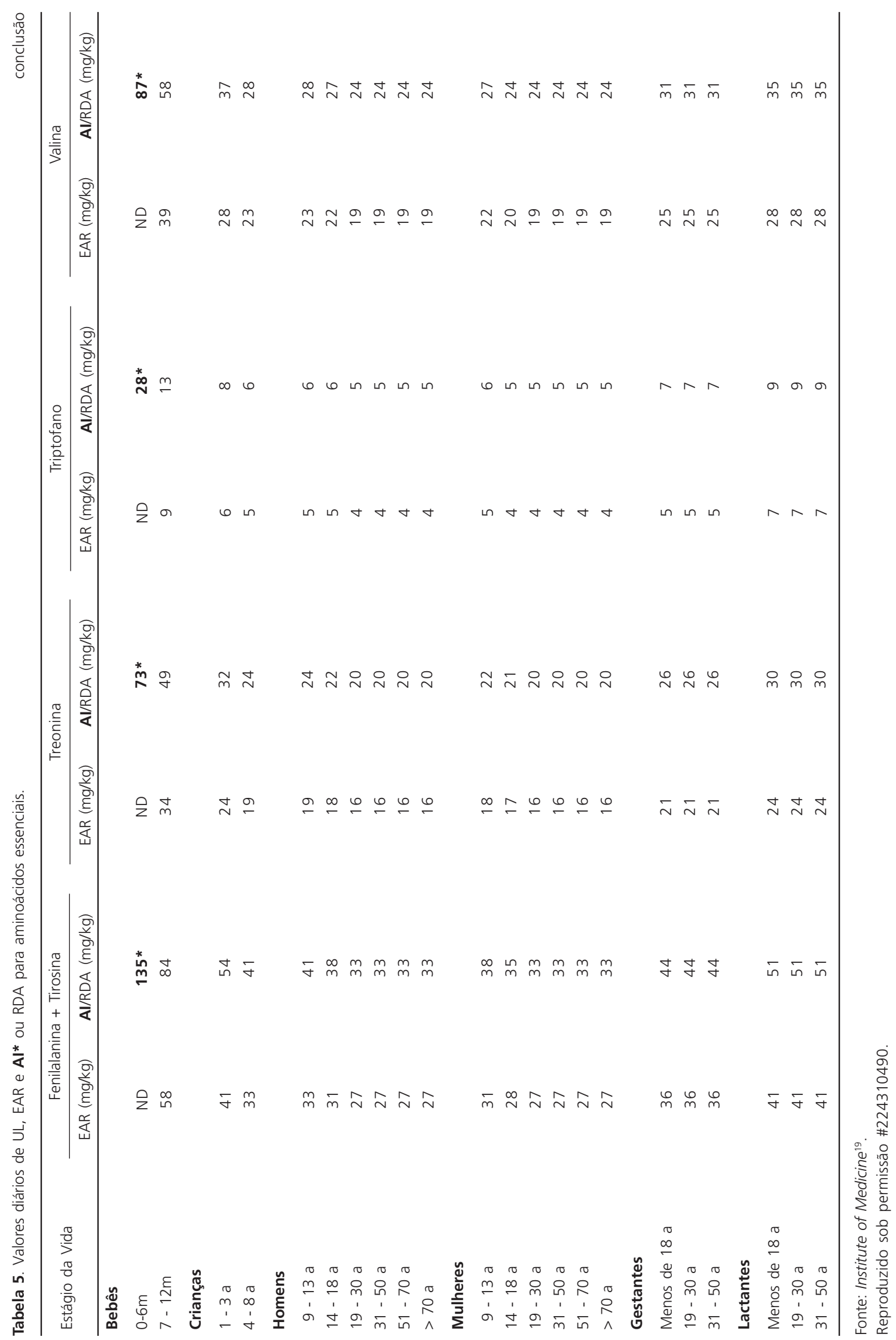


758 | R.M. PADOVANI et al.

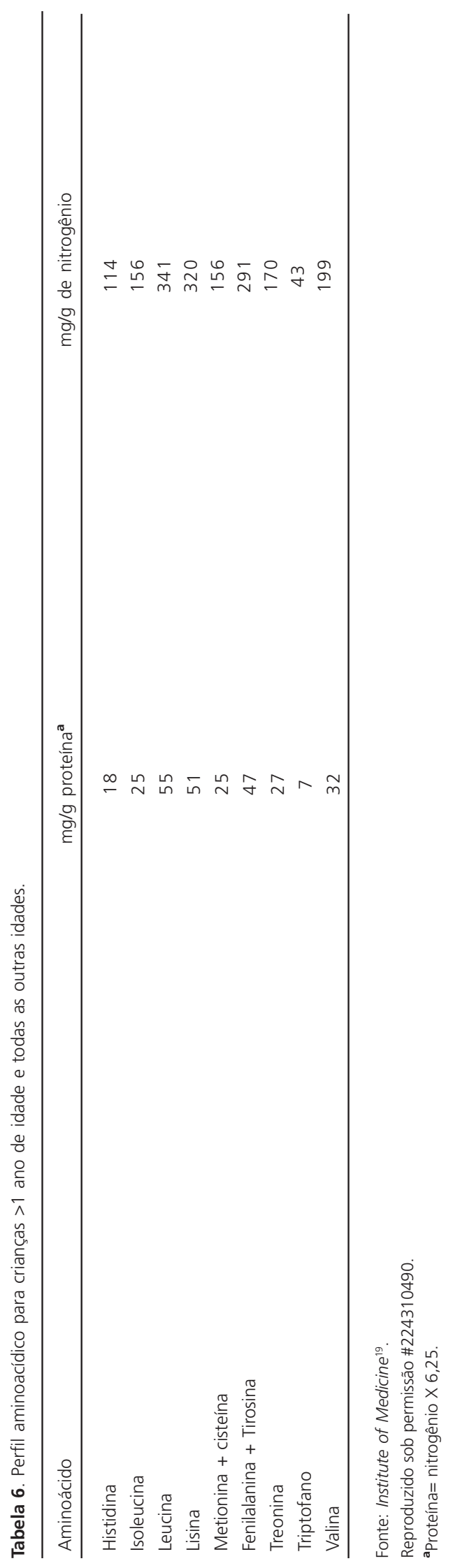




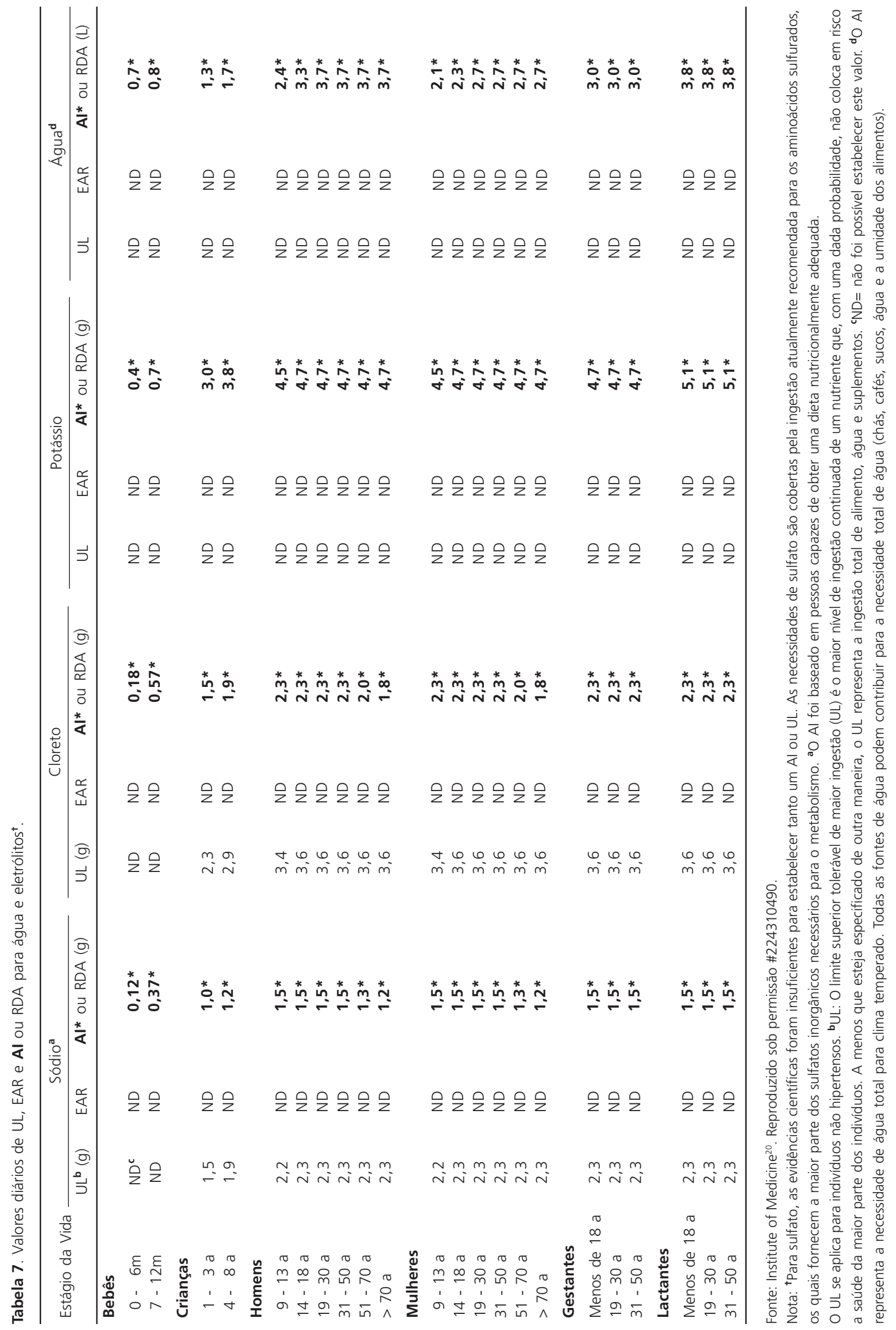




\section{REFERÊ NCIAS}

1. Hegsted DM. Establishment of nutritional requirements in man. Bordens Rev Nutr Res. 1959; 20(2):13-22.

2. Institute of Medicine. Dietary reference intakes for calcium, phosphorus, magnesium, vitamin $D$, and fluoride. Washington (DC): National Academy Press; 1997.

3. Food and Agriculture Organization of the United Nations. Human energy requirements. Rome, 2004. Report of a Joint FAO/WHO/UNU Expert Consultation Food and Nutrition Technical report series 1. [cited 2006 Mar 1]. Available from: http://www.fao.org/documents/show_htm

4. Hauber U, Neuhauser-Berthold M. Historical development of dietary recommendations. Z Ernährungswiss. 1996; 35(2):157-62.

5. Institute of Medicine. Dietary reference intakes: a risk assessment model for establishing upper intake levels for nutrients. Washington (DC): National Academy Press; 1998.

6. Food and Agriculture Organization of the United Nations. Human vitamin and mineral requirements. Rome; 2002. Report of a joint FAOMHO expert consultation Bangkok.

7. Institute of Medicine. Dietary reference intakes: applications in dietary planning. Washington (DC): National Academy Press; 2003.

8. Institute of Medicine. Dietary reference intakes: applications in dietary assessment. Washington (DC): National Academy Press; 2000.

9. Institute of Medicine. Dietary reference intakes for vitamin $C$, vitamin $E$, selenium, and carotenoids. Washington (DC): National Academy Press; 2000.

10. Nusser SM, Carriquiry AL, Dood KW, Fuller WA. A semiparametric transformation approach to estimating usual daily intake distribuitions. J Am Stat Assoc. 1996; 91(436):1440-9.

11. Institute of Medicine. Dietary reference intakes for thiamin, riboflavin, niacin, vitamin B6, folate, vitamin B12, pantothenic acid, biotin, and choline. Washington (DC): National Academy Press; 1998.
12. Institute of Medicine. Dietary reference intakes for vitamin A, vitamin K, arsenic, boron, chromium, copper, iodine, iron, manganese, molybdenum, nickel, silicon, vanadium, and zinc. Washington (DC): National Academy Press; 2002.

13. Trumbo P, Yates AA, Schlicker S, Poos M. Dietary reference intakes: vitamin $A$, vitamin $K$, arsenic, boron, chromium, copper, iodine, iron, manganese, molybdenum, nickel, silicon, vanadium, and zinc. J Am Diet Assoc. 2001; 101(3):294-301.

14. Slater B, Marchioni DL, Fisberg RM. Estimando a prevalência da ingestão inadequada de nutrientes. Rev Saúde Pública. 2004; 38(4):599-605.

15. Carriquiry AL. Assessing the prevalence of nutrient inadequacy. Public Health Nutr. 1999; 2(1):23-33.

16. National Research Council. Nutrient adequacy: assessment using food consumption surveys. Washington (DC): National Academy Press; 1986.

17. Amaya-Farfán J, Domene SMA, Padovani RM. DRIs. Síntese comentada das novas propostas sobre recomendações nutricionais para antioxidantes. Rev Nutr. 2001; 14(1):71-8.

18. Marchioni DML, Slater B, Fisberg RM. Aplicação das dietary reference intakes na avaliação da ingestão de nutrientes para indivíduos. Rev Nutr. 2004; 17(2):207-16.

19. Institute of Medicine. Dietary reference intakes for energy, carbohydrate, fiber, fat, fatty acids, cholesterol, protein, and amino acids. Washington (DC): National Academy Press; 2005.

20. Institute of Medicine. Dietary reference intakes for water, potassium, sodium, chloride, and sulfate. Washington (DC): National Academy Press; 2004.

21. Institute of Medicine. Dietary reference intakes; the essential guide to nutriente requirements. Washington (DC): National Academy Press; 2006.

Recebido em: 3/6/2006

Versão final reapresentada em: 11/8/2006 Aprovado em: 30/9/2006 